\title{
BLANCHET, PHILIPPE (2017): LES MOTS PIÉGÉS DE LA POLITIQUE. PARIS: TEXTUEL. 112 PÁGINAS
}

\author{
EGUZKI URTEAGA \\ Universidad Del País Vasco \\ eguzki.urteaga@ehu.eus
}

Philippe Blanchet acaba de publicar su último libro titulado Les mots piégés de la politique en la editorial Textuel. Conviene recordar que el autor, catedrático de sociolingüística en la Universidad Rennes 2, es responsable del Master internacional "Francofonía, Plurilingüísmo y Mediación intercultural”, co-director de los Cahiers Internationaux de Sociolinguistique y redactor jefe de los Cahiers de Linguistique, revue de sociolinguistique de langue française. Sus investigaciones se centran en tres ámbitos: el análisis de la complejidad de la pluralidad lingüística y cultural en contextos francófonos; la didáctica de la pluralidad lingüística y de las relaciones interculturales; las políticas lingüísticas y educativas y las discriminaciones lingüísticas. Ese trabajo ha dado lugar a numerosos libros, entre los cuales podemos citar Langues, cultures et identités régionales en Provence. La Métaphore de l'aïoli (2002), Linguistique de terrain, méthode et théorie. Une approche ethno-sociolinguistique de la complexité (2012) o Discriminations: combattre la glottophobie (2016).

La presente obra empieza con una amplia introducción en la cual Blanchet subraya que « el poder no se conquista y no se ejerce únicamente en la esfera material. No es solamente una cuestión de política institucional y militar, de fuerza jurídica y política o de [recursos] económicos y financieros. Se conquista y se ejerce también en las actividades del lenguaje, aquellas por las cuales los [seres] humanos organizan el mundo y le dan sentido poniéndolo en palabras" (p.9). En ese sentido, "el poder es una cuestión de lengua, discurso y vocabulario. Se ejerce difundiendo e imponiendo (...) sentidos implícitos, presupuestos [y] prejuicios, que [se inscriben] en los usos de ciertas palabras [y] en (...) ciertos discursos" (p.9). Como lo subrayan Luc Boltanski y Pierre Bourdieu, “el poder sobre la lengua es una de las dimensiones más importantes del poder" (Boltanski y Bourdieu, 1975: 12).

De hecho, los que detienen el poder necesitan obtener el consentimiento de aquellos sobre los cuales lo ejercen. "Necesitan que los dominados consientan a su propia dominación, que acepten un cierto orden social para que esa dominación y ese orden, para que los privilegios de los [favorecidos], no sean cuestionados" (pp.9-10). Como lo indica el autor, "es por la puesta en palabras, por su generalización, gracias a una propaganda sutil (...)

Para citar esta reseña / To cite this book review: Urteaga, Eguzki (2017). Reseña de Philippe Blanchet (2017): Les mots piégés de la politique. Paris: Textuel (112 páginas) ELUA, 31: 377-382. doi:10.14198/ELUA2017.31.21

Enlace / Link: http://dx.doi.org/10.14198/ELUA2017.31.21 
que se [ejerce] esa dominación consentida que [se] denomina hegemonía. Esa hegemonía [mantiene] un cierto orden social imponiendo la creencia absoluta en una concepción única de la sociedad que [se] denomina ideología" (p.10). En ese sentido, "la cuestión no es tanto la de las ideas como la de la hegemonía de las ideas por (...) su puesta en palabras” (p.11).

Precisamente, este libro tiene como objetivo poner de manifiesto los contenidos implícitos de términos "insertados en discursos engañosos. Se trata de desvelar ese adoctrinamiento por el cual un pensamiento se convierte en hegemónico y por el cual una ideología es discretamente impuesta a un gran número [de personas]" (p.11). La ideología puesta de manifiesto por Blanchet alude a cierta identidad política y nacional francesa. "Esa ideología impone una única versión de lo que sería la República” (p.11). Para ilustrar su tesis, el autor ha elegido una serie de palabras significativas, utilizadas con frecuencia, que traducen ese discurso: laicidad, radicalización, comunitarismo e incivilidades (p.11).

A ese respecto, Blanchet indica que "la comunicación política se basa cada vez más en elementos de lenguaje" (p.13). Estos elementos de lenguaje "tienen como objeto manipular (...) la agenda mediática e imponer una interpretación de los acontecimientos" (Alduy, 2016: 47). En ese sentido, "rechazar la invasión de los discursos por los nuevos sentidos insidiosos de los nuevos usos de estas palabras, que imponen implícitamente unas maneras de pensar, [y] hacer fracasar las trampas semánticas e ideológicas, es encontrar de nuevo la libertad de expresión y la confianza en su derecho a [tomar] la palabra, en su propio pensamiento, en su propia lucha, en su propia visión del mundo [y] en su propio proyecto de sociedad" (p.14). Conviene estar atento a todo ello "para denunciar los abusos pero también para actuar mejor" (p.14), ya que, a través de ese vocabulario, el poder fija el marco en el cual se produce el debate, se actúa y se construye una sociedad determinada. De hecho, "para cambiar la sociedad, hay que cambiar de marco y, por lo tanto, cambiar de palabras" (p.14).

Empezando su análisis por la palabra "laicidad", Blanchet recuerda que ese término "ha aparecido en 1870, en el momento en el cual se preparaban las leyes Ferry sobre la escuela pública (1882-1886) y, posteriormente, la ley de separación de [las Iglesias] y el Estado (1905)" (p.35). No en vano, se observa desde el inicio del nuevo milenio un fuerte aumento de la utilización de ese término, especialmente en los medios de comunicación (p.35). Ese auge coincide con los atentados del 11 de septiembre de 2001 en Nueva York, el debate surgido en Francia sobre el velo islámico, la aprobación de la ley sobre la prohibición de los signos religiosos ostentosos en los centros educativos en 2004, la publicación de caricaturas del profeta Mahoma en un semanario galo, el debate suscitado por Nicolas Sarkozy sobre la inmigración y la identidad nacional francesa, la prohibición del velo islámico integral en el espacio público o, más recientemente, la polémica en torno al uso del burkini en las playas del Hexágono (p.36). La mediatización de estos acontecimientos aparece como "una reacción masiva, exacerbada y en caliente a acontecimientos variados, vinculados de manera diversa a la religión musulmana. (...) Se trata (...) de una reacción de oposición al Islam y no de una política de fondo sobre las relaciones razonadas entre las instituciones, la sociedad civil y las religiones" (p.36).

En su origen, el término "laicidad" designa un principio político y jurídico según el cual ninguna religión tiene el monopolio del Estado y de la vida social y según el cual el Estado y sus instituciones no privilegian a ninguna religión, las respetan todas y garantizan la libertad de conciencia y de religión de cada uno (p.37). "Es el sentido generalmente admitido de la ley de 1905" (p.37). En ese sentido, la ley de 2004 introduce un cambio al respecto, puesto 
que pasa "de la neutralidad del Estado y de los servicios públicos a una neutralidad impuesta a los usuarios de estos servicios, [es decir] a los alumnos de los centros educativos" (p.37). Esto significa que desaparece la separación entre la esfera de la autoridad estatal y la esfera de las libertades individuales que estaba claramente marcada hasta entonces (p.38). "Una vez esta forma de separación borrada (...), la puerta [está] abierta a otras neutralizaciones" (p.38). Por lo cual, la laicidad se ha convertido en uno de los valores centrales de la República, a la vez "indiscutible e intangible" (p.39). Así, en la "libreta del ciudadano" entregado a las personas candidatas a la adquisición de la nacionalidad francesa, se menciona la laicidad como un valor esencial de la República (pp.41-42).

De manera general, el término "laicidad" ha pasado de una semántica de la acogida a una semántica de la prohibición (p.42). En ese sentido, se produce una radicalización de la laicidad por dos vías: por una parte, transformando una noción inclusiva y no-discriminatoria en una noción que excluye y discrimina; $y$, por otra parte, dejando entender, e incluso imponiendo a través del discurso, que solo habría una acepción de la laicidad erigida en valor primordial y, por lo tanto, no discutible (p.43). Esto significa que "la nueva laicidad promovida desde los años 2000 en Francia es [presentada] como un valor sacralizado, como un dogma indiscutible, es decir como una creencia religiosa, una fe laica. Esta concepción de la laicidad se convierte en hegemónica" (p.44). Como lo subraya el autor, la laicidad es recuperada y reciclada, a contrasentido de su significado original, "en el proceso general de sacralización patriótica e identitaria que afecta a Francia" (p.45). Existe, por lo tanto, "una laicidad discriminatoria, la que [ataca] a la religión y la que, simultáneamente, constituye el marco de otra creencia dogmática" (p.46).

Además, varios estudios de psicología social (Kamiejski et al., 2012; Nugier et al, 2016) han mostrado que "el nuevo significado y [la nueva centralidad] concedida a la noción de laicidad han tenido efectos graves, modificando los comportamientos de las personas que viven en Francia en sus relaciones [sociales]" (p.48). Incide de dos maneras. Por una parte, convirtiendo la laicidad en una dimensión fundamental de la organización de la sociedad francesa. Por otra parte, acentuando notablemente los discursos y las actitudes negativas hacia las personas de origen magrebí en comparación con otros ciudadanos galos y hacia los musulmanes en comparación con los católicos (p.48).

Como lo subraya Blanchet, esta concepción y esta práctica de la laicidad son fuentes de discriminación: 1) han sido desarrolladas principalmente en reacción a la presencia de musulmanes y de prácticas musulmanas; 2) solo conciernen a aquellos cuya religión se manifiesta en la esfera pública, a través de su vestimenta por ejemplo; 3) excluyen del sistema educativo a personas, vulnerando sus derechos fundamentales y propiciando su marginación en lugar de favorecer su inclusión; 4) incrementan las discriminaciones, dado que los musulmanes residentes en Francia son mayoritariamente de origen magrebí o subsahariano; y, 5) contribuyen a la normalización e incluso a una forma de legitimación de la xenofobia en Francia (pp.49-50). Por lo tanto, nos dice el autor, "estamos ante una inversión de un término y de una noción, instrumentalizados y ahogados en [una serie] de discursos auto-legitimadores por supuestos valores patrióticos, en beneficio de una ideología nacionalista [y] de una política de exclusión, discriminación [y] exacerbación de los conflictos” (p.50). Si la laicidad protegía en el pasado, se ha convertido en un peligro para ciertos colectivos (p.50).

La segunda palabra estudiada es la de "radicalización". Esta noción, y sus derivados "radicalizar" y "radicalizado", han sido utilizados de manera masiva por los responsables 
políticos y los medios de comunicación (p.53). "Prácticamente inexistente en la prensa durante los años 1990, se desarrolla a partir de los años $2000(\ldots)$, y la frecuencia de su tratamiento mediático es multiplicada por cuatro a partir de $2011 \mathrm{y}$, sobre todo, de 2014, especialmente a propósito del Islam [y] del terrorismo (...) islamista” (p.53). Y, "los crímenes cometidos en 2015 y 2016 en nombre del Daech han aumentado (...) los usos de manera exponencial" (p.53). Conviene señalar, al respecto, que su uso es dos veces más frecuente en los periódicos de derechas que en los de izquierdas (p.53). Igualmente ausente de las políticas públicas hasta 2014, se ha convertido en uno de los aspectos fundamentales de los planes de acción del gobierno galo, especialmente en la justicia y la educación, de cara a "prevenir y detectar la radicalización" (p.53).

En su acepción política, la palabra "radicalización", derivada del verbo "radicalizar" aparecido en los años 1930, sirve para "nombrar el hecho de radicalizar(se), especialmente en relación con el Partido Radical y, más ampliamente, con movimientos revolucionarios de izquierdas. Es únicamente a partir de los años $2000 \mathrm{y}$, sobre todo, 2010 cuando es utilizada en su sentido actual, a la vez mucho más amplio (...) y mucho más caracterizado" (pp.55). De hecho, los documentos oficiales difundidos por las instituciones, en sus acciones "de prevención y detección de la radicalización”, realizan amalgamas en torno a la noción de "radicalización”. Así, la radicalización deja de ser estrictamente violenta, extremista y terrorista, y la atención se centra exclusivamente en una "radicalización musulmana potencialmente yihadista" (pp.56-57).

Según Blanchet, "radicalización" no es un término adecuado por varias razones. "En primer lugar, porque el campo político designa históricamente [con esa palabra] a progresistas humanistas completamente opuestos a los oscurantistas religiosos (...). En segundo lugar, porque engloba [hasta tal punto] que sus utilizadores y promotores principales no consiguen ni definirlo de manera clara ni ponerse de acuerdo entre ellos. En tercer lugar, porque deja entender que toda radicalidad es en sí peligrosa (...). En cuarto lugar, porque su uso no especificado pero implícitamente reservado a un fanatismo religioso preciso crea a la vez estigma e injusticia para las poblaciones concernidas por esa religión (...). En quinto lugar, porque deja pensar que los fanáticos en cuestión solo aplican su religión de manera intransigente e intensa" (pp.60-61).

Asimismo, el término "comunitarismo" forma parte de las palabras que circulan masivamente en la esfera mediática y que traducen posiciones políticas determinadas. "Funciona en una oposición binaria y, por lo tanto, simplista: republicano [versus] comunitarista" (p.69). "El comunitarismo sería la reivindicación de derechos específicos por minorías divergentes del modelo francés, sean lingüísticas, culturales [o] religiosas, y que querrían [convertirse] en comunidades intermedias entre los individuos que las componen y la comunidad nacional francesa" (p.69). En realidad, el término "comunitarista" es empleado para designar y estigmatizar a la comunidad arabo-musulmana que representaría una amenaza para la República gala (p.69).

A ese respecto, Fabrice Dhum-Sonzogni (2016) demuestra que, inicialmente, la palabra "comunitarista" es un neologismo francés que ha entrado en los diccionarios a partir de 1997 y cuyo uso es relativamente escaso hasta los años noventa del pasado siglo, antes de extenderse rápidamente con el inicio del nuevo milenio (pp.71-72). En ese sentido, hemos pasado de un uso reducido en los años 1990, centrado básicamente en la organización de la comunidad europea, a un uso notable en la década siguiente, asociado a cuestiones de 
seguridad. Por último, en los años 2010, su utilización se ha generalizado en un contexto marcado por la "normalización del referencial nacionalista" (p.72). Dhum-Sonzogni pone de manifiesto cómo "toda una serie de usos presentan el término (...) como una evidencia, sin cuestionarlo jamás” (pp.72-73).

Para ese investigador, el discurso crítico hacia un supuesto comunitarismo pretende hablar en nombre de los valores republicanos, que son la libertad, la igualdad y la fraternidad, cuando, en la práctica, los vulnera. "Aspira a reducir la libertad de las personas que pertenecen supuestamente a estas comunidades (...); mantener y reforzar las desigualdades que padecen (...), construyendo categorías diferentes de la población; [y] romper la fraternidad, excluyendo a estas personas de la solidaridad global" (p.76).

Por su parte, el término "incivilidad" tiene principalmente el sentido de mala educación, falta de respeto hacia los demás, malos modales y escasa cortesía. "Construido a partir de la palabra 'civilidad', está presente desde hace varios siglos pero su frecuencia en cuanto al uso [ha sido] muy baja. En realidad, [se trata de] una palabra rara hasta los años 2010" (p.86). La supuesta multiplicación de las incivilidades ha dado lugar al desarrollo de la doctrina de la "tolerancia cero" hacia ellas. Esta doctrina, y las medidas a las que ha dado lugar, son a menudo discriminatorias, generan una mayor agresividad y son fuente de conflictos (p.89). Prueba de esta excesiva seguridad, "la ley del 22 de marzo de 2016 relativa a la prevención y lucha contra las incivilidades, los ataques a la seguridad pública y los actos terroristas en los transportes colectivos de pasajeros, asocia las incivilidades y el terrorismo" (p.89). Para Blanchet, "la evolución de la sociedad francesa hacia [una neutralización] verbal se manifiesta cada vez más desde hace dos (...) décadas" (p.90). Forma parte de un proceso general de desarrollo de un control social y de una reducción de las libertades fundamentales en una sociedad cada vez más desigual (p.90).

En la conclusión de su libro, el autor recuerda que "el estudio de estas palabras-clave, que ocupan un lugar central en los discursos políticos y en sus [traducciones] mediáticas, permite confirmar un deslizamiento general de la sociedad francesa, al menos de su concepción de la sociedad, hacia un modelo nacionalista, autoritario, uniformizador y xenófobo" (p.97). Ese deslizamiento se manifiesta por cambios en los "efectos de sentido" producidos por los usuarios de estas palabras, "tanto para aquellos que los difunden como para aquellos que los reciben" (p.97). Las palabras laicidad, radicalización, comunitarismo o incivilidades son buena prueba de ello. Más ampliamente, el autor coincide plenamente con Pierre Bourdieu que ha puesto de manifiesto el poder de las palabras en sus usos (p.98). De hecho, "todos los discursos son mantenidos a partir de un punto de vista. Todos revelan, explícitamente o implícitamente, una idea preconcebida, unas orientaciones [y] unas convicciones. Todos contienen necesariamente implícitos y presupuestos" (p.102).

Al término de la lectura de Les mots piégés de la politique, es obvio reconocer el amplio recorrido y la larga aportación del autor al análisis del discurso político, situándose en la filiación de Jean-Baptiste Marcellesi (1971) y Pierre Bourdieu (1982). Asimismo, hace gala de pertinencia y lucidez en su análisis de nociones que son utilizadas, a menudo, de manera interesada e incluso malintencionada, traicionando su significado original y siendo objeto de asociaciones cuestionables. No duda, para ello, en ilustrar sus tesis con ejemplos concretos, provenientes de la actualidad, y en utilizar un lenguaje accesible que convierte la lectura de su libro en agradable. No en vano, y de cara a matizar esta valoración positiva, a veces se deja llevar por su pasión por el objeto de estudio y sus sensibilidades sociolingüísticas y 
políticas, lo que lo conduce a utilizar superlativos que son innecesarios dada la claridad de la exposición y la fundamentación empírica de la argumentación.

En cualquier caso, la lectura de esta obra es recomendable en un contexto político y mediático marcado por un uso ambiguo y, a veces, abusivo de ciertas palabras.

\section{Referencias bibliográficas}

Alduy, C. (2016): "La rhétorique politique entre conviction et intoxication", Sciences Humaines, $\mathrm{n}^{\circ} 287$.

Blanchet, P. (2002): Langues, cultures et identités régionales en Provence. La Métaphore de l'aïoli. París: L'Harmattan.

Blanchet, P. (2012): Linguistique de terrain, méthode et théorie. Une approche ethno-sociolinguistique de la complexité, édition revue et complétée. Rennes: Presses Universitaires de Rennes.

Blanchet, P. (2016): Discriminations: combattre la glottophobie. París: Textuel.

Blanchet, P. (2017): Les mots piégés de la politique. París: Textuel.

Boltanski, L. y Bourdieu, P. (1975): «Le fétichisme de la langue », Actes de la Recherche en Sciences Sociales, ${ }^{\circ} 4$.

Bourdieu, P. (1982): Ce que parler veut dire, l'économie des échanges linguistiques. París: Fayard.

Dhum-Sonzogni, F. (2016): Communautarisme. Enquête sur une chimère du nationalisme français. París: Demopolis.

Kamiekski, R. et al. (2016): «Le modèle républicain d'intégration: implications pour la psychologie des relations entre groupes ", L’Année psychologique, n¹12, pp. 51-85.

Marcellesi, J-P. (1971): Le Congrès de Tours. Etudes sociolinguistiques. París: Le Pavillon.

Nugier, A. et al. (2016): « Nouvelle laïcité en France et pression normative envers les minorités musulmanes », International Review of Social Psychology, n²9-1, pp. 15-30. 